\title{
SIMPLY CONNECTED SPACES
}

\author{
BY \\ ROBERT S. NOVOSAD
}

The two definitions of a simply connected space in general use are

(1) A connected, locally connected space $X$ is simply connected if every covering space of $X$ is isomorphic to the trivial covering space of $X$.

(2) An arcwise connected space $X$ is simply connected if every closed path in $X$ is homotopic to a constant path at some point $x$ of $X$.

It is shown in [4] that these definitions are not equivalent.

We see that (1) restricts $X$ to the class of locally connected spaces. Moreover, the definition of the fundamental group of a space $Y$ in terms of the simply connected covering space of $Y$ (see page 52 of [1]) is applicable only when $Y$ is connected, locally connected, and locally simply connected inasmuch as the existence of simply connected covering spaces is known only for this class of spaces. On the other hand (2) has the disadvantage that several basic theorems do not hold true for such simply connected spaces, the most important being the principle of monodromy.

The purpose of this paper is to give a definition of simple connectedness which will not be subject to the objections listed above. This is accomplished by generalizing the concept of covering space. For spaces which are locally arcwise connected and locally simply connected these generalized covering spaces are covering spaces of the usual sort.

Theorem (2.6) will show that every arcwise connected space $X$ has a universal generalized covering space $\tilde{X}$ which is simply connected under the new definition. We shall use $\tilde{X}$ to define a fundamental group of $X$. Theorem (3.2) is the principle of monodromy.

1. Covering spaces. We modify a property of covering spaces given by proposition (1) on page 50 of [1] to extend the notion of covering space. We replace the simply connected space of this proposition with a contractible space inasmuch as it is our intention to define simple connectedness.

(1.1) Definition. Let $X$ be an arcwise connected space and let $x_{0} \in X$. A triple $\left(Y, y_{0}, p\right)$ consisting of an arcwise connected space $Y$, a point $y_{0} \in Y$, and a mapping

$$
p:\left(Y, y_{0}\right) \rightarrow\left(X, x_{0}\right)
$$

will be called a generalized covering space (abbreviated g-covering space) if given any contractible space $A$, a point $a_{0} \in A$, which is a deformation retract of $A$, and a map

Received by the editors July 1, 1954. 


$$
\alpha:\left(A, a_{0}\right) \rightarrow\left(X, x_{0}\right),
$$

then there exists a map

$$
\tilde{\alpha}:\left(A, a_{0}\right) \rightarrow\left(Y, y_{0}\right)
$$

which is unique with respect to the property $p \tilde{\alpha}=\alpha$.

The notation $X$ for an arcwise connected space and $X_{0}^{t}$ for the space of paths in $X$ with initial point $x_{0}$ and having the compact open topology will be standard throughout the paper. Also $x_{0}^{f}$ will stand for the constant path at the point $x_{0}$. All spaces will be Hausdorff spaces. Connectedness will mean Hausdorff-Lennes connectedness. The projection

$$
\lambda:\left(X_{0}^{t}, \underset{x_{0}}{t}\right) \rightarrow\left(X, x_{0}\right)
$$

will be the one which sends a path $f$ onto the point $f(1)$ of $X$. Given any map $q:\left(Z, z_{0}\right) \rightarrow\left(X, x_{0}\right)$, the induced map

$$
q_{\ddagger}:\left(Z_{0}^{*}, z_{0}^{*}\right) \rightarrow\left(X_{0}^{*}, x_{0}^{*}\right)
$$

will always be the usual one, $q_{t}(f)$ being the path $f$ followed by the mapping $q$.

The letter $A$ will always denote a contractible space and the letter $I$ will stand for the unit interval $[0,1]$. Given a contraction

$$
H: A \times I \rightarrow A
$$

of $A$ onto a point $a_{0}$ with $a_{0}$ held fixed during the homotopy, the map

$$
h:\left(A, a_{0}\right) \rightarrow\left(A_{0}^{\ddagger}, a_{0}^{\ddagger}\right),
$$

defined for any $a \in A$ and $t \in I$ by $h(a)(t)=H(a, t)$, is continuous (see Theorem 1 of [3]) and is one to one.

(1.3) Lemma. Let $\left(Y, y_{0}, p\right)$ be a g-covering space of $\left(X, x_{0}\right)$. Then the map induced on the space of paths

$$
p_{\sharp}:\left(Y_{0}^{*}, y_{0}^{\dagger}\right) \rightarrow\left(X_{0}^{*}, x_{0}^{*}\right)
$$

is a homeomorphism onto.

Proof. The fact that a path is a mapping of the contractible space $I$ into $X$ leads immediately to the fact that $p_{r}$ is one to one and onto.

Now $\left(X_{0}^{t}, x_{0}^{t}\right)$ is contractible with respect to the mapping $H$ defined for any $f \in X_{0}^{t}$ and $s, t \in I$ by

$$
H(f, t)(s)=f(t s) .
$$

Hence the projection $\lambda:\left(X_{0}^{f}, x_{0}^{f}\right) \rightarrow\left(X, x_{0}\right)$ can be lifted to a map

$$
\rho:\left(X_{0}^{\dagger}, x_{0}^{\dagger}\right) \rightarrow\left(Y, y_{0}\right)
$$


for which $\lambda=p \rho$. In turn $\rho$ induces a map

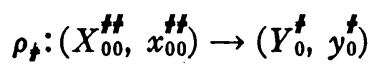

where $X_{00}^{f f}$ denotes the space of paths of $X_{0}^{f}$ with initial point $x_{0}^{f}$, and $x_{00}^{\# f}$ is the constant path at $x_{0}^{f}$. The contraction $H$ is constant on the point $x_{0}^{f}$ so we get a one to one map

$$
h:\left(X_{0}^{+}, x_{0}^{\dagger}\right) \rightarrow\left(X_{00}^{\#}, x_{00}^{\#+}\right)
$$

as given by (1.2).

We have for any $f \in X_{0}^{t}$

$$
p_{t} \rho_{\boldsymbol{f}} h(f)=p \cdot \rho \cdot h(f)=\lambda \cdot h(f)=h(f)(1)=H(f, 1)=f .
$$

Hence $p_{t} \rho_{t} h$ is the identity mapping of $X_{0}^{t}$, and $p_{t}^{-1}=\rho_{t} h$ is continuous.

It is easy to prove the following lemma directly from the definition of $g$ covering space.

(1.4) Lemma. Let $\left(Y, y_{0}, p\right)$ be a g-covering space of $\left(X, x_{0}\right)$, let $x_{1}$ be any point of $X$, and let $y_{1}$ be any point of $p^{-1}\left(x_{1}\right)$. Then $\left(Y, y_{1}, p\right)$ is a $g$-covering space of $\left(X, x_{1}\right)$.

We note that the set $p^{-1}\left(x_{1}\right)$ mentioned above is always totally arcwise disconnected or else the constant path at $x_{1}$ would not be only the image of the constant path at $y_{1}$ under the map $p_{t}$.

(1.5) Lemma. In the diagram

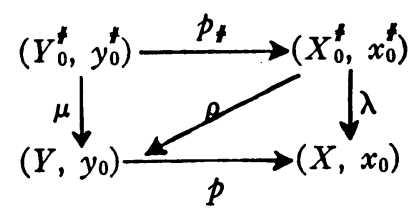

let $p$ be a mapping of an arcwise connected space $Y$ into $X$, and let $\lambda$ and $\mu$ be the usual projections. If $\rho$ is any continuous map of $X_{0}^{f}$ onto $Y$ for which $\lambda=p \rho$ and either

(a) $\rho p_{t}=\mu$ or

(b) $p^{-1}(x)$ is totally arcwise disconnected for each $x \in X$ and $p_{+}$is one to one, then $\left(Y, y_{0}, p\right)$ is a g-covering space of $\left(X, x_{0}\right)$.

Proof. Given a mapping of a contractible space

$$
\alpha:\left(A, a_{0}\right) \rightarrow\left(X, x_{0}\right)
$$

we have the induced mapping

$$
\alpha \neq:\left(A_{0}^{f}, a_{0}^{f}\right) \rightarrow\left(X_{0}^{f}, x_{0}^{f}\right) .
$$


Moreover $\lambda \alpha+h=\alpha$ where $h$ is given by (1.2). Then

$$
\rho \alpha_{f} h:\left(A, a_{0}\right) \rightarrow\left(Y, y_{0}\right)
$$

is such that $p \rho \alpha_{t} h=\lambda \alpha_{\sharp} h=\alpha$.

Suppose that hypothesis (a) holds and that

$$
\beta:\left(A, a_{0}\right) \rightarrow\left(Y, y_{0}\right)
$$

is any map for which $p \beta=\alpha$. Then $p_{t} \beta_{t}=\alpha+$ where

$$
\beta_{f}:\left(A_{0}^{t}, a_{0}^{\dagger}\right) \rightarrow\left(Y_{0}^{\dagger}, y_{0}^{\prime}\right)
$$

is induced by $\beta$, and

$$
\beta=\mu \beta_{t} h=\rho p_{t} \beta_{t} h=\rho \alpha_{t} h .
$$

Thus $\rho \alpha+h$ is unique and $\left(Y, y_{0}, p\right)$ is a g-covering space of $\left(X, x_{0}\right)$.

Now there are two maps

$$
\nu, \lambda_{f}:\left(X_{00}^{H}, x_{00}^{H+}\right) \rightarrow\left(X_{0}^{f}, x_{0}^{f}\right)
$$

defined on any path $F:(I, 0) \rightarrow\left(X_{0}^{f}, x_{0}^{*}\right)$ and $t \in I$ by

$$
\begin{aligned}
\nu(F)(t) & =F(1)(t), \\
\lambda_{f}(F)(t) & =\lambda F(t)=F(t)(1) .
\end{aligned}
$$

The fact that $F(0)(t)=x_{0}^{t}(t)=x_{0}=F(t)(0)$ for all $t \in I$ leads to an easy proof that $\nu(F)$ and $\lambda_{f}(F)$ are paths in $X$ with the same end points $x_{0}$ and $F(1)(1)$, and that they are homotopic to each other with the end points held fixed throughout the homotopy. This means that $\nu(F)$ and $\lambda_{f}(F)$ are in the same arc-component of $\lambda^{-1}(F(1)(1))$. Clearly $\lambda \lambda_{f}(F)=F(1)(1)=\lambda \nu(F)$.

Assume that hypothesis (b) holds. Suppose $g, g^{\prime} \in X_{0}^{f}$ are such that

$$
\lambda g=\lambda g^{\prime}=x
$$

for some $x \in X$ but that $\rho g \neq \rho g^{\prime}$. Then $\rho g$ and $\rho g^{\prime}$ are different elements of the totally arcwise disconnected set $p^{-1}(x)$, and therefore $g$ and $g^{\prime}$ do not lie in the same component of $\lambda^{-1}(x)=\rho^{-1} p^{-1}(x)$.

If hypothesis (a) does not hold, then there exists $f \in Y_{0}^{f}$ such that

$$
\mu(f) \neq \rho p_{f}(f) .
$$

We can show that the induced mapping

$$
\rho_{f}:\left(X_{00}^{\prime \prime}, x_{00}^{\prime \prime}\right) \rightarrow\left(Y_{0}^{\prime}, y_{0}^{\prime}\right)
$$

is also onto. Let $F \in X_{00}^{\#}$ be such that $\rho_{f}(F)=f$. Then

$$
\rho \lambda_{f}(F)=\rho p_{f} \rho_{f}(F)=\rho p_{f}(f) \neq \mu(f)=\mu \rho_{f}(F)=\rho_{f}(F)(1)=\rho F(1)=\rho \nu(F) .
$$


We have arguments above which show $\lambda_{f}(F)$ and $\nu(F)$ to be both in and not in the same component of $\lambda^{-1}(F(1)(1))=\lambda^{-1}(\nu F(1))=\lambda^{-1}\left(\lambda_{t} F(1)\right)$. Thus hypothesis (a) is a consequence of (b). This ends the proof of (1.5).

We shall say that a map $p:\left(Y, y_{0}\right) \rightarrow\left(X, x_{0}\right)$ covers $X$ evenly if for every point $x \in X$ there is an open set $U$ containing $x$ and such that $p^{-1}(U)$ is the union of disjoint open sets $\left\{V_{j}\right\}$ each of which is mapped homeomorphically onto $U$ by $p$. Then any path $f \in X_{0}^{f}$ can be lifted in the usual way to a path $\tilde{f} \in Y_{0}^{*}$ which is unique with respect to the property

$$
p_{r}(\tilde{f})=p \cdot \tilde{f}=f \text {. }
$$

It follows that $p t$ is one to one and onto.

We refer to the diagram of (1.5) and consider the function

$$
\rho:\left(X_{0}^{*}, x_{0}^{*}\right) \rightarrow\left(Y, y_{0}\right)
$$

given by $\rho=\mu p^{-1}$. We see that

$$
p \rho=p \mu p_{t}^{-1}=\lambda p_{t} p_{t}^{-1}=\lambda .
$$

Let $V$ be an open set of $Y$ mapped homeomorphically by $p$. Then

$$
\rho^{-1}(V)=\rho^{-1} p^{-1} p(V)=\lambda^{-1} p(V)
$$

is an open set of $X_{0}^{*}$. Now it is clear that the open sets $V$ which are mapped homeomorphically by $p$ form a basis for the open sets of $Y$, and so $\rho$ is continuous.

Denote the arc-component of $y_{0}$ in $Y$ by $c Y$. Clearly $Y_{0}^{*}=(c Y)_{0}^{*}$, and $\mu$ maps $Y_{0}^{f}$ onto $c Y$. We define

$$
\rho^{\prime}:\left(X_{0}^{\dagger}, x_{0}^{\dagger}\right) \rightarrow\left(c Y, y_{0}\right)
$$

by setting $\rho^{\prime}(f)=\rho(f)=\mu p_{f}^{-1}(f)$. We see that $\rho^{\prime}$ is onto, continuous, $\rho^{\prime} p \neq=\mu$, and $(p \mid c Y) \rho^{\prime}=\lambda$. Then (1.5) (a) applied to $c Y$ yields

(1.6) Lemma. Let $p:\left(Y, y_{0}\right) \rightarrow\left(X, x_{0}\right)$ cover $X$ evenly, and let $c Y$ be the arccomponent of $y_{0}$ in $Y$. Then $\left(c Y, y_{0}, p \mid c Y\right)$ is a g-covering space of $X$.

2. The universal g-covering space. The proof of the following lemma is trivial.

(2.1) Lemma. If $\left(Z, x_{0}, q\right)$ is a g-covering space of $\left(Y, y_{0}\right)$, and $\left(Y, y_{0}, p\right)$ is a $g$-covering space of $\left(X, x_{0}\right)$, then $\left(Z, z_{0}, p q\right)$ is a g-covering space of $\left(X, x_{0}\right)$.

(2.2) Definition. Given g-covering spaces $\left(Y, y_{0}, p\right)$ and $\left(Z, z_{0}, q\right)$ of $\left(X, x_{0}\right)$ we shall write

$$
\left(Y, y_{0}, p\right) \geqq\left(Z, z_{0}, q\right)
$$


if $\left(Y, y_{0}, r\right)$ is a g-covering space of $\left(Z, z_{0}\right)$ where $r:\left(Y, y_{0}\right) \rightarrow\left(Z, z_{0}\right)$ is such that $q r=p$.

We note that the map $r$ is unique, otherwise the process which lifts a mapping of a contractible space $A$ into $X$ to a mapping of $A$ into $Z$ would not be unique. It is clear that we obtain a bona fide equivalence relation from

(2.3) Definition. If $\left(Y, y_{0}, p\right) \geqq\left(Z, z_{0}, q\right)$ and $\left(Z, z_{0}, q\right) \geqq\left(Y, y_{0}, p\right)$ we say that $\left(Y, y_{0}, p\right)$ and $\left(Z, z_{0}, q\right)$ are equivalent $g$-covering spaces of $\left(X, x_{0}\right)$.

If $\left(Y, y_{0}, p\right)$ and $\left(Z, z_{0}, q\right)$ are equivalent g-covering spaces of $\left(X, x_{0}\right)$ there exist unique maps

$$
s:\left(Z, z_{0}\right) \rightarrow\left(Y, y_{0}\right), \quad r:\left(Y, y_{0}\right) \rightarrow\left(Z, z_{0}\right)
$$

such that $p s=q$ and $q r=p$. Then $\left(Y, y_{0}\right)$ is a g-covering space of itself with respect to the mapping $s r$ for which

$$
p s r=q r=p .
$$

Since the identity mapping $i_{y}$ of $Y$ is the unique mapping having this property we have $s r=i_{y}$. Similarly we have $r s=i_{z}$. It follows that $r$ is a homeomorphism of $Y$ onto $Z$ and that $s$ is its inverse. Incidentally if we tried to develop the theory without using the base points $x_{0}, y_{0}$, and $z_{0}$, sr would not necessarily be the identity map of $Y$ and the following lemma would not be true.

(2.4) Lemma. $A$ set $L$ which contains one g-covering space of $\left(X, x_{0}\right)$ from each equivalence class and contains all the covering mappings of these spaces onto one another forms an inverse system of spaces and mappings (i.e. $L$ is a directed set with respect to the relation $\geqq$ and, given $\left(Y, y_{0}, p\right) \geqq\left(Z, z_{0}, q\right) \geqq\left(W, w_{0}, u\right)$ in $L$, the covering maps $s:\left(Y, y_{0}\right) \rightarrow\left(Z, z_{0}\right), t:\left(Z, z_{0}\right) \rightarrow\left(W, w_{0}\right)$, and $r:\left(Y, y_{0}\right)$ $\rightarrow\left(W, w_{0}\right)$ are such that $\left.t s=r\right)$.

Proof. That $L$ is partially ordered by $\geqq$ follows from the fact that $L$ contains but one representative of each equivalence class. The trivial g-covering space $\left(X, x_{0}, i_{x}\right)$ is a lower bound of $L$, and we shall take it to be the representative of its class in $L$, hence $L$ is directed. With $s, t$, and $r$ as above, each is unique and since $u(t s)=p$ as well as $u r=p$ it follows that $t s=r$.

(2.5) Definition. Let $\tilde{X}$ be the inverse limit of the system $L$ and let $\tilde{x}_{0}$ be the point $\left(\cdots, x_{0}, \cdots, y_{0}, \cdots\right)$ determined by the base points of the spaces in $L$. Given any $\left(Y, y_{0}, p\right)$ in $L$ let

$$
F_{y}:\left(\tilde{X}, \tilde{x}_{0}\right) \rightarrow\left(Y, y_{0}\right)
$$

be the projection onto $Y$ (i.e. $F_{y}$ is the restriction to $\tilde{X}$ of the projection $F_{y}^{\prime}$ of the cartesian product $P L=P\left[Z:\left(Z, z_{0}, q\right) \in L\right]$ onto $\left.Y\right)$.

(2.6) Theorem. Any g-covering space $\left(Y, y_{0}, p\right)$ of $\left(X, x_{0}\right)$ has $\left(\tilde{X}, \tilde{x}_{0}, F_{y}\right)$ as a g-covering space. 
Proof. It is clear we may assume $\left(Y, y_{0}, p\right)$ to be in $L$. Let

$$
\beta:\left(A, a_{0}\right) \rightarrow\left(Y, y_{0}\right)
$$

map a contractible space into $Y$. Then

$$
p \beta:\left(A, a_{0}\right) \rightarrow\left(X, x_{0}\right),
$$

and given $\left(Z, z_{0}, q\right)$ in $L$ there exists a unique

$$
\beta_{z}:\left(A, a_{0}\right) \rightarrow\left(Z, z_{0}\right)
$$

such that $q \beta_{z}=p \beta$.

We have a mapping into the cartesian product of the sets of $L$

$$
B:\left(A, a_{0}\right) \rightarrow\left(P L, \bar{x}_{0}\right)
$$

determined as follows. Given $a \in A$ the $Z$ component of $B(a)$ is $\beta_{z}(a)=F_{z}^{\prime} B(a)$ where $F_{z}^{\prime}$ is the projection of $P L$ onto $Z$. Now if $\left(W, w_{0}, u\right)$ is another member of $L$ and

$$
r:\left(Z, z_{0}\right) \rightarrow\left(W, w_{0}\right)
$$

is a covering mapping in $L$, we have $q=u r$, and

$$
r F_{z}^{\prime} B=F_{w}^{\prime} B=\beta_{w}:\left(A, a_{0}\right) \rightarrow\left(W, w_{0}\right)
$$

since

$$
u r F_{z}^{\prime} B=q F_{z}^{\prime} B=q \beta_{z}=u \beta_{w}=u F_{w}^{\prime} B
$$

and no map $\beta^{\prime} \neq \beta_{w}$ is such that $u \beta^{\prime}=u \beta_{w}$. Thus for any $a \in A, B(a) \in \tilde{X} \subset P L$. Also $F_{z}^{\prime} B\left(a_{0}\right)=\beta_{z}\left(a_{0}\right)=z_{0}$ for all $Z \in L$ so $B\left(a_{0}\right)=\tilde{x}_{0}$.

We define

$$
\tilde{\beta}:\left(A, a_{0}\right) \rightarrow\left(\tilde{X}, \tilde{x}_{0}\right)
$$

by $\tilde{\beta}(a)=B(a)$. Then

$$
F_{y} \tilde{\beta}=F_{y}^{\prime} B=\beta .
$$

Also $F_{z} \tilde{\beta}=F_{z}^{\prime} B=\beta_{z}$ is unique for all $Z$, and since $\tilde{\beta}$ is determined by its components, $\tilde{\beta}$ is unique with respect to ${ }^{*}$ above. This ends the proof.

In view of this lemma we are justified in calling $\left(\tilde{X}, \tilde{x}_{0}, F_{x}\right)$ a universal $g$-covering space of $\left(X, x_{0}\right)$. We shall call a space $W$-simply connected if every g-covering space of $\left(W, w_{0}\right)$ is equivalent to the trivial one for some $w_{0} \in W$. Clearly $\tilde{X}$ is g-simply connected.

The group of homeomorphisms $\theta: \tilde{X} \rightarrow \tilde{X}$ for which $F_{x} \theta=F_{x}$ will be called the $g$-fundamental group $\pi g_{1}(X)$ of $X$. Unlike the theory for locally simply connected spaces, here we may have a space whose g-fundamental group is trivial but which is not simply connected. For example take $W$ to be the graph of the equation 


$$
y=\sin (\pi / x),
$$$$
0<x \leqq 1,
$$

in a Euclidean plane in which we identify the points $(0,0)$ and $(1,0)$. Then the space $\tilde{W}$ is a half open interval, and $F_{w}$ is a one to one mapping which is not a homeomorphism. This space is also an example of a simply connected space as defined in terms of paths which does not satisfy the principle of monodromy.

There exist spaces on which the g-fundamental group differs from the ordinary fundamental group and also from the topologized fundamental group of Hurewicz [2].

It is not hard to show that there is a one to one correspondence between the elements of $\pi g_{1}(X)$ and the points of $F_{x}^{-1}\left(x_{0}\right)$ in $\tilde{X}$. The topology imparted to $\pi g_{1}(X)$ by this correspondence is not necessarily discrete. Both $\pi g_{1}(X)$ and its topology can be shown to be homotopy invariants of the space $X$. Multiplication by a fixed factor and the taking of inverses are continuous in $\pi g_{1}(X)$, however a rather complicated example can be given in which $\pi g_{1}(X)$ is not a topological group.

It is sometimes easy to see whether or not this topology is discrete even though it is not easy to calculate any of the ordinary group invariants. In this way the topology may be useful in checking the homotopy types of spaces.

3. Simply connected spaces. The following is another basic property (at. least as it regards covering spaces) which is not enjoyed by spaces which are simply connected as defined in terms of paths.

(3.1) Theorem. Let $\left(Y, y_{0}, p\right)$ be a g-covering space of $\left(X, x_{0}\right)$. Then given a map

$$
\beta:\left(W, w_{0}\right) \rightarrow\left(X, x_{0}\right)
$$

of a g-simply connected space $W$ into $X$, there exists a unique map

$$
\beta_{1}:\left(W, w_{0}\right) \rightarrow\left(Y, y_{0}\right)
$$

for which $p \beta_{1}=\beta$.

Proof. The mappings $\lambda, \mu, \nu, \beta_{t}$, and $p t$ of the diagram below follow our standard notation, and $p \neq$ is a homeomorphism by (1.3).

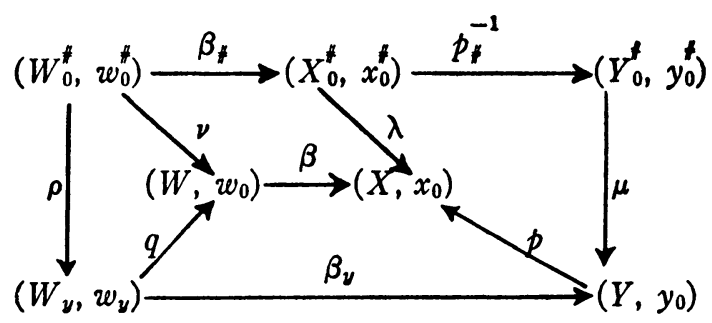


Let us say that elements $f ; g \in W_{0}^{*}$ are $Y$-equivalent if both

(a) $\nu(f)=\nu(g)($ i.e. $f(1)=g(1))$,

(b) $\mu p_{*}^{-1} \beta_{f}(f)=\mu p_{*}^{-1} \beta_{*}(g)$.

We denote the $Y$-equivalence class of $f$ by $[f]$. Then the set of all such equivalence classes is represented by the symbol $W_{y}$, and $\rho$ denotes the natural mapping $\rho(f)=[f]$. We impose the quotient space topology on $W_{\nu}$ (i.e. $O \subset W_{\nu}$ is open if and only if $\rho^{-1}(O)$ is open in $W_{0}^{*}$ ).

The mapping $q$ of the diagram is defined on any $[f] \in W_{y}$ by

$$
q[f]=f(1)=\nu(f) .
$$

Clearly $q \rho=\nu$. In view of (a) above, $q$ is well defined. If $U$ is an open set of $W$ then $\nu^{-1}(U)=\rho^{-1} q^{-1}(U)$ is open in $W_{0}^{*}$, hence $q^{-1}(U)$ is open and $q$ is continuous.

The mapping $\beta_{y}$ of the diagram is defined by

$$
\beta_{y}[f]=\mu p^{-1} \beta_{*}(f) \text {. }
$$

Clearly $\beta_{y} \rho=\mu p_{t}^{-1} \beta_{\sharp}$. Also $p \beta_{y} \rho=p \mu p_{t}^{-1} \beta_{\sharp}=\lambda \beta_{\sharp}=\beta \nu=\beta q \rho$, and since $\rho$ is onto we have

$$
p \beta_{y}=\beta q .
$$

We show that $\beta_{y}$ is well defined and continuous by the same method that we used above for $q$.

Now for any $w \in W$

$$
p \beta_{y} q^{-1}(w)=\beta q q^{-1}(w)=\beta(w)
$$

and we see that $\beta_{y}$ maps $q^{-1}(w)$ into the set $p^{-1} \beta(w)$. If $[f]$ and $[g]$ are points of $q^{-1}(w)$ such that

$$
\beta_{y}[f]=\beta_{y}[g],
$$

we have both (a) and (b) above holding for $f$ and $g$. Thus $[f]=[g]$, and we see that $\beta_{y}$ maps $q^{-1}(w)$ in a one to one fashion into the set $p^{-1} \beta(w)$ which is totally arcwise disconnected for all $\beta(w), w \in W$. Consequently $q^{-1}(w)$ is totally arcwise disconnected. Since $W_{0}^{*}$ is arcwise connected so is $W_{y}=\rho W_{0}^{*}$.

It follows from $(1.5)(\mathrm{b})$ that $\left(W_{y}, w_{y}, q\right)$ is a g-covering space of $\left(W, w_{0}\right)$. Now $W$ is a simply connected space so $q$ must be a homeomorphism. We set

$$
\beta_{1}=\beta_{y} q^{-1}:\left(W, w_{0}\right) \rightarrow\left(Y, y_{0}\right)
$$

and get

$$
p \beta_{1}=p \beta_{y} q^{-1}=\beta q q^{-1}=\beta
$$

as desired.

Now suppose $\beta_{2}:\left(W, w_{0}\right) \rightarrow\left(Y, y_{0}\right)$ is such that 


$$
p \beta_{2}=\beta=p \beta_{1} .
$$

Then we have, for the maps induced on the spaces of paths,

$$
p+\beta_{2 f}=p+\beta_{1}
$$

and since $p+$ is a homeomorphism,

$$
\beta_{2 f}=\beta_{1 f}
$$

Then

$$
\lambda \beta_{2 f}=\lambda \beta_{1 f}
$$

and by commutativity

$$
\beta_{2} \nu=\beta_{1} \nu .
$$

Since $\nu$ is onto we get $\beta_{2}=\beta_{1}$ which completes the proof of the theorem.

We alter the principle of monodromy to make it refer to g-simply connected spaces.

(3.2) TheOREM. Let $W$ be a g-simply connected space and let $E$ be any nonempty set. Assume that to every point $(u, v)$ of some fixed arcwise connected open neighborhood $D$ of the diagonal of $W \times W$ there is assigned a function

$$
\phi_{u v}:(u) X_{\imath} E \rightarrow(v) \times_{s} E
$$

where $X$. denotes the cartesian product of sets. We further assume that for $u, v, w \in W$

(1) $\phi_{u v}$ is one to one and onto,

(2) $\phi_{u u}$ is the identity function,

(3) if $\phi_{u v}, \phi_{v w}$, and $\phi_{u w}$ are defined, $\phi_{u i v}=\phi_{w v} \phi_{v w}$.

Then there exists a set function

$$
\psi: W \rightarrow W \times, E
$$

such that

(a) for any $w \in W, \psi(w) \in(w) \times_{8} E$,

(b) if $\phi_{u v}$ is defined, $\psi(v)=\phi_{u v} \psi(u)$,

(c) given any $w_{0} \in W$ and given any $\left(w_{0}, e_{0}\right) \in\left(w_{0}\right) X_{8} E$ we may select $\psi$ in such a way that $\psi\left(w_{0}\right)=\left(w_{0}, e_{0}\right)$, and this assignment of $\psi\left(w_{0}\right)$ uniquely determines $\psi$.

Proof. For $w \in W$, for any open set $N$ containing $w$ such that $N \times N \subset D$, and for $e \in E$ we have the subset

$$
U((w, e), N)=\left[\phi_{w u}(w, e): u \in N\right]
$$

of $W \times_{s} E$. The process used on p. 48 of [1] can be used here to show that the sets $U((w, e), N)$ (written $U(w, N, e)$ in [1]) form a basis for the open sets of a topology on $W \times, E$. We denote the resulting space by $Z$. The map 


$$
\omega: Z \rightarrow W
$$

is the projection, $\omega(w, e)=w$. Proofs given in [1] show

(A) $\omega$ maps each $U((w, e), N)$ homeomorphically onto $N$,

(B) for $(w, e) \neq\left(w^{\prime}, e^{\prime}\right)$ there exist open neighborhoods $N$ of $w$ and $N^{\prime}$ of $w^{\prime}$ such that $U\left(\left(w^{\prime}, e^{\prime}\right), N^{\prime}\right)$ and $U((w, e), N)$ are disjoint. For $w=w^{\prime}$ we can take $N=N^{\prime}$.

Given $w_{0} \in W$ we take any $z_{0}=\left(w_{0}, e_{0}\right)$. We see that

$$
\omega:\left(Z, z_{0}\right) \rightarrow\left(W, w_{0}\right)
$$

covers $W$ evenly. It follows from (1.6) that $\left(c Z, z_{0}, c \omega\right)$ is a g-covering space of $\left(W, w_{0}\right.$ ) when $c Z$ denotes the arc component of $z_{0}$ in $Z$ and $c \omega=\omega \mid c Z$ is the restriction of $\omega$ to $c Z$.

Since $W$ is g-simply connected, $c \omega$ must be a homeomorphism. The function

$$
\psi: W \rightarrow W \times{ }_{8} E
$$

is defined for any $w$ by

$$
\psi(w)=c \omega^{-1}(w) .
$$

We see that conclusion (a) of our theorem holds and proceed with the proof of (b). Given any $(u, v) \in D$ let

$$
\beta:(I, 0) \rightarrow\left(D,\left(w_{0}, w_{0}\right)\right)
$$

be a path for which $\beta(1)=(u, v)$. Let $\left\{M_{i}\right\}$ and $\left\{N_{i}\right\}, i=1, \cdots, n$, be two families of open sets of $W$ such that:

(i) The family $\left\{M_{i} \times N_{i}\right\}$ forms an open cover of $\beta(I)$.

(ii) Each of the sets $M_{i} \times M_{i}, M_{i} \times N_{i}$, and $N_{i} \times N_{i}$ is contained in $D$.

(iii) There exist points $0=t_{0}<t_{1}<\cdots<t_{n-1}<t_{n}=1$ such that the closed interval $\left[t_{i-1}, t_{i}\right]$ is mapped by $\beta$ into $M_{i} \times N_{i}$.

It is clear that (b) holds for the point $\left(w_{0}, w_{0}\right)=\beta\left(t_{0}\right)$. Let us suppose that $\beta\left(t_{i-1}\right)=(w, x)$ is such that

$$
\psi(x)=\phi_{w x}(\psi(w)) .
$$

If we set $(r, s)=\beta\left(t_{i}\right)$ we see that both $r$ and $w$ are in $M_{i}$. The set $U\left(\psi(w), M_{i}\right)$ contains the point $\phi_{w r} \psi(w)$ and no other point with $r$ as first coordinate since it is projected homeomorphically onto $M_{i}$.

Let $\Sigma: D \rightarrow W$ be defined at any point $\left(w_{1}, w_{2}\right) \in D$ by $\Sigma\left(w_{1}, w_{2}\right)=w_{1}$. It follows from (iii) above that $r=\Sigma \beta\left(t_{i}\right)$ and $w=\Sigma \beta\left(t_{i-1}\right)$ are connected by an arc in $M_{i}$, hence $\psi(w)$ and $\phi_{w r} \psi(w)$ are connected by an arc in $U\left(\psi(w), M_{i}\right)$. Then $\phi_{w r} \psi(w)$ is contained in the arc-component of $\psi(w)$ which is $c Z$. We have

$$
\phi_{w r} \psi(w)=c \omega^{-1}(r)=\psi(r) .
$$


Similarly we get

$$
\phi_{x 8} \psi(x)=\psi(s) .
$$

All the mappings in the following equations are defined because of (ii) above.

$\psi(s)=\phi_{x s} \psi(x)=\phi_{x s} \phi_{w x} \psi(w)=\phi_{w s} \psi(w)=\phi_{w s} \phi_{r w} \phi_{w r} \psi(w)=\phi_{r s} \phi_{w r} \psi(w)=\phi_{r s} \psi(r)$.

Now (b) follows by induction on $i$.

The proof of the uniqueness of $\psi$ given on page 49 of [1] may be applied here to finish our proof.

For the purpose of distinguishing the types of simple connectedness we shall say that an arcwise connected space $X$ is $A$-simply connected if all its closed paths are null homotopic, and we shall say that $X$ is C-simply connected if it has no nontrivial covering spaces. First we prove

(3.3) Lemma. Let $\left(Y, y_{0}, p\right)$ be a covering space of a space $\left(Z, z_{0}\right)$, and let $c Y$ and $c Z$ denote the arc-components of $y_{0}$ in $Y$ and $z_{0}$ in $Z$ respectively (according to the definition of covering space as given in [1], $Y$ and $Z$ must be connected but need not be arcwise connected). Then $\left(c Y, y_{0}, p \mid c Y\right)$ is a g-covering space of $\left(c Z, z_{0}\right)$. Moreover the map

$$
p_{f}:\left(Y_{0}^{\ddagger}, y_{0}^{\ddagger}\right) \rightarrow\left(Z_{0}^{\ddagger}, z_{0}^{\dagger}\right)
$$

induced on the space of paths is a homeomorphism onto.

Proof. Clearly $p$ is such that $p^{-1}(c Z)$ covers $c Z$ evenly. It follows from (1.6) that the arc-component $c p^{-1}(c Z)$ of $y_{0}$ in $p^{-1}(c Z)$ forms a $g$-covering space of $c Z$. Since $p(c Y) \subset c Z$ we have $c Y \subset c p^{-1}(c Z) \subset c Y$. Then $c Y=c p^{-1}(c Z)$, and $\left(c Y, y_{0}, p \mid c Y\right)$ is a g-covering space of $\left(c Z, z_{0}\right)$.

Clearly $c Y_{0}^{*}=Y_{0}^{*}$ and $c Z_{0}^{*}=Z_{0}^{*}$. The last statement of the lemma now follows directly from (1.3).

(3.4) Corollary. A locally connected, g-simply connected space $\left(Z, z_{0}\right)$ is C-simply connected.

Proof. The definition of g-simple connectedness entails arcwise connectedness so $Z=c Z$. Then we have from (3.3) that $\left(c Y, y_{0}, p \mid c Y\right)$ is a g-covering space of $\left(Z, z_{0}\right)$ and must be trivial by hypothesis. Hence $p \mid c Y$ is a homeomorphism of $c Y$ onto $Z$, and this can only happen if $c Y=Y$, in other words only if $Y$ is a trivial covering space of $Z$.

(3.5) THEOREM. Let $X$ be an arcwise connected, locally arcwise connected, and locally $A$-simply connected space. Then the following are equivalent:

(1) $X$ is C-simply connected.

(2) $X$ is A-simply connected.

(3) $X$ is g-simply connected. 
Proof. The covering space $\tilde{X}$ constructed from the space of paths (see [2]) is an A-simply connected covering space of $X$. If (1) above holds, $X$ and $\tilde{X}$ are homeomorphic and (2) follows.

Now suppose that (2) holds and that $\left(Y, y_{0}, p\right)$ is a g-covering space of $\left(X, x_{0}\right)$. It can be shown that $p$ is an open map under the restrictions of the theorem, and therefore that $p$ is not one to one unless $\left(Y, y_{0}, p\right)$ is a trivial g-covering space of $\left(X, x_{0}\right)$.

If $y_{1} \neq y_{2}$ are points of $Y$ such that $p\left(y_{1}\right)=p\left(y_{2}\right)$ let $f_{e}$ be a path in $Y$ from $y_{0}$ to $y_{\epsilon}, \epsilon=1,2$. It is easy to show that $p_{t}\left(f_{1}\right)$ and $p_{t}\left(f_{2}\right)$ are nonhomotopic paths in $X$ from $x_{0}$ to $p\left(y_{1}\right)$-a contradiction to (2). Hence (2) implies (3). That (1) follows from (3) is a consequence of (3.4).

The condition that $X$ be locally A-simply connected can be weakened somewhat; however some such condition is needed to show that (2) follows from either (1) or (3). This is the only place that it is used in the proof. It seems probable that the equivalence of (1) and (3) can be proved under conditions weaker than those given.

\section{REFERENCES}

1. Claude Chevalley, Theory of Lie groups, Princeton University Press, 1946.

2. J. Dugundji, A topologized fundamental group, Proc. Nat. Acad. Sci. U.S.A. vol. 36 (1950) pp. 141-143.

3. R. H. Fox, On topologies for function spaces, Bull. Amer. Math. Soc. vol. 51 (1945) pp. 429-432.

4. Tudor Ganea, Simply connected spaces, Fund. Math. vol. 38 (1951) pp. 179-203.

The Pennsylvania State University

University PARK, Pa. 